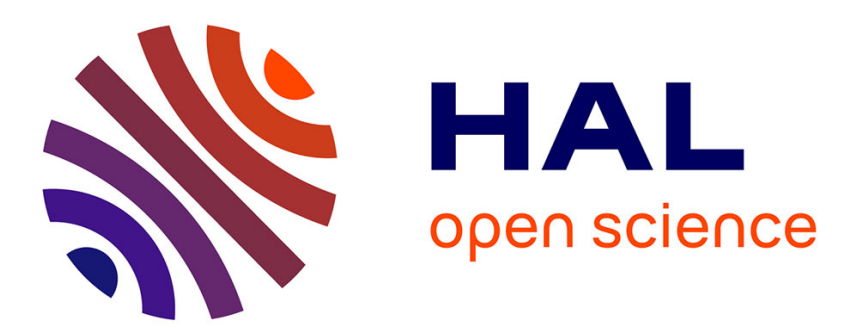

\title{
A stable recursive state estimation filter for models with nonlinear dynamics subject to bounded disturbances
}

\author{
Yasmina Becis-Aubry, Mohamed Boutayeb, Mohamed Darouach
}

\section{To cite this version:}

Yasmina Becis-Aubry, Mohamed Boutayeb, Mohamed Darouach. A stable recursive state estimation filter for models with nonlinear dynamics subject to bounded disturbances. 45th IEEE Conference on Decision and Control, CDC'06, Dec 2006, San Diego, Californie, United States. pp.CDROM. hal-00122107

\section{HAL Id: hal-00122107 \\ https://hal.science/hal-00122107}

Submitted on 26 Dec 2006

HAL is a multi-disciplinary open access archive for the deposit and dissemination of scientific research documents, whether they are published or not. The documents may come from teaching and research institutions in France or abroad, or from public or private research centers.
L'archive ouverte pluridisciplinaire HAL, est destinée au dépôt et à la diffusion de documents scientifiques de niveau recherche, publiés ou non, émanant des établissements d'enseignement et de recherche français ou étrangers, des laboratoires publics ou privés. 


\title{
A stable recursive state estimation filter for models with nonlinear dynamics subject to bounded disturbances
}

\author{
Y. Becis-Aubry, M. Boutayeb and M. Darouach
}

\begin{abstract}
This contribution proposes a recursive and easily implementable online algorithm for state estimation of multi-output discrete-time systems with nonlinear dynamics and linear measurements in presence of unknown but bounded disturbances corrupting both the state and measurement equations. The proposed algorithm is based on state bounding techniques and is decomposed into two steps : time update and observation update that uses a switching estimation Kalman-like gain matrix. Particular emphasis is given to the design of a weighting factor that ensures consistency of the estimated state vectors with the input-output data and the noise constraints and that guarantees the stability of the algorithm.
\end{abstract}

\section{INTRODUCTION}

State estimation of stochastic dynamical systems has been extensively studied during the last decades and the problem is usually solved by assuming white and Gaussian noises on model and measurements (Kalman filter). However, when the statistical properties of the noises are unknown or not satisfied, an alternative approach consists in considering that only bounds on the possible magnitude of the disturbances are available, the so-called set-membership estimation was first introduced by Schweppe [1] using ellipsoidal bounding techniques. The aim is to determine a set of state estimate vectors compatible with the bounds on the process disturbance and measurement noise. Since these pioneer works, a vast literature is dedicated to this subject in the context of state estimation of linear systems [2], [3], [4], [5], [6]. Some interesting algorithms have been developed for nonlinear models like most of real-life problems : [7], [8], [9] [10], but very few in the ellipsoidal context. The technique used here has also been developed for the identification of nonlinear MIMO models [11] and the state estimation of linear models [12].

The goal of this paper is to outline a recursive algorithm for state estimation of discrete-time systems with nonlinear dynamics and linear measurements in presence of unknown but bounded disturbances corrupting both the state and measurement vectors.

The paper is organized as follows. In the first section, the problem is formulated and the aims of the designed algorithm are set out. Then the two stages of the algorithm are detailed successively : the second section presents the time update step that computes the predicted estimate and the predicted ellipsoid containing all possible values of the state; the third section details the observation update step which uses a switching estimation Kalman-like gain matrix with a weighting parameter that ensures consistency of the estimated state vectors with the input-output data and the noise constraints. In the forth section, some properties of the algorithm are established, especially, those expressed in the aims and the Input to State stability is proved. Finally, the effectiveness of the developed algorithm is demonstrated through a numerical example.determine a progression law for the ellipsoid $\mathcal{E}_{k}:=\mathcal{E}\left(\hat{\boldsymbol{x}}_{\boldsymbol{k}}, \sigma_{k}^{2} P_{k}\right)$

Notations : (i) For a square matrix $M, M \succ 0_{s \times n}$ (resp. $M \succeq 0_{s \times n}$ ) means that $M \in \mathbb{R}^{s \times n}$ is symmetric and positive definite (resp. semidefinite); (ii) $\mathcal{E}(c, P):=\left\{x \in \mathbb{R}^{s} \mid(x-c)^{T} P^{-1}(x-c) \leq 1\right\}$ is an ellipsoid in $\mathbb{R}^{s}\left(s \in \mathbb{N}^{*}\right)$, where $c \in \mathbb{R}^{s}$ is its center and $P \in \mathbb{R}^{s \times s}$ is a symmetric positive definite matrix that defines its shape, size and orientation in the $\mathbb{R}^{s}$ space; (iii) $\|\boldsymbol{x}\|=\left(\boldsymbol{x}^{T} \boldsymbol{x}\right)^{\frac{1}{2}}$ is the Euclidean norm of the vector $\boldsymbol{x}$ and $\|\boldsymbol{x}\|_{W}=\left(\boldsymbol{x}^{T} W \boldsymbol{x}\right)^{\frac{1}{2}}$ is its weighted Euclidean norm ( $W \succ 0$ of appropriate dimension); (iv) $0_{n \times m}$ denotes a zero matrix of $\mathbb{R}^{n \times m}$ and $\mathbf{0}$ denotes a zero vector of appropriate dimension; (v) $\lambda_{i}(M)$ denotes the $i^{\text {th }}$ eigenvalue of a square matrix $M, \operatorname{tr}(M)=\sum_{i} \lambda_{i}(M)$ is its trace and $\operatorname{det}(M)=\prod_{i} \lambda_{i}(M)$ is its determinant; (vi) the symbol := means that the RHS is defined to be equal to the LHS.

\section{Problem formulation}

Let us consider the following discrete-time system written in the state space :

$$
\begin{aligned}
\boldsymbol{x}_{\boldsymbol{k}}^{*} & =\varphi\left(\boldsymbol{x}_{\boldsymbol{k}-1}^{*}, \boldsymbol{u}_{k-1}\right)+\boldsymbol{w}_{k-1} \\
\boldsymbol{y}_{\boldsymbol{k}} & =F_{k} \boldsymbol{x}_{\boldsymbol{k}}^{*}+\boldsymbol{v}_{\boldsymbol{k}}
\end{aligned}
$$

where $\boldsymbol{x}_{\boldsymbol{k}}^{\boldsymbol{*}} \in \mathbb{R}^{n}$ is the unknown state vector to be estimated; $\boldsymbol{u}_{\boldsymbol{k}} \in \mathbb{R}^{m}$ is the control input vector $; \boldsymbol{y}_{\boldsymbol{k}} \in \mathbb{R}^{p}(p<n)$ is a measurable system output vector $; F_{k} \in \mathbb{R}^{p \times n}$ is the output matrix of full row rank and $\boldsymbol{w}_{\boldsymbol{k}} \in \mathbb{R}^{n}$ and $\boldsymbol{v}_{\boldsymbol{k}} \in \mathbb{R}^{p}$ are unobservable bounded noise vectors with unknown statistical characteristics that may include modeling inaccuracies,

Y. Becis-Aubry is with Université d'Orléans, LVR UPRES-A 7038. 63 av. de Lattre de Tassigny 18000 Bourges, FRANCE. Yasmina.Becis@bourges.univ-orleans.fr

M. Boutayeb is with Université Louis Pasteur - Strasbourg 1, LSIIT - CNRS. Bd Sébastien Brant Illkirch, 67400 Strasbourg FRANCE.

M. Darouach is with Université Henri Poincaré - Nancy 1, CRAN UMR 7039 CNRS. 186 rue de Lorraine, 54400 Cosnes et Romain, FRANCE. 
discretization errors or computer round-off errors. $\boldsymbol{v}_{\boldsymbol{k}}$ can represent the measurement noise and $\boldsymbol{w}_{\boldsymbol{k}-\mathbf{1}}$ can be viewed as unknown but bounded inputs. The only properties verified by $\boldsymbol{v}_{\boldsymbol{k}}$ and $\boldsymbol{w}_{\boldsymbol{k}-\boldsymbol{1}}$ are

$$
\begin{aligned}
\boldsymbol{w}_{\boldsymbol{k}} \in \mathcal{E}\left(\mathbf{0}, W_{k}\right) & \Leftrightarrow \quad \boldsymbol{w}_{\boldsymbol{k}}^{T} W_{k}^{-1} \boldsymbol{w}_{\boldsymbol{k}} \leq 1, \forall k \in \mathbb{N}^{*} \\
\boldsymbol{v}_{\boldsymbol{k}} \in \mathcal{E}\left(\mathbf{0}, V_{k}\right) & \Leftrightarrow \boldsymbol{v}_{\boldsymbol{k}}^{T} V_{k}^{-1} \boldsymbol{v}_{\boldsymbol{k}} \leq 1, \forall k \in \mathbb{N}^{*} . r
\end{aligned}
$$

The function $\varphi_{k}: \mathbb{R}^{n} \longrightarrow \mathbb{R}^{n}, \boldsymbol{x} \longmapsto \boldsymbol{\varphi}_{\boldsymbol{k}}(\boldsymbol{x}):=\varphi\left(\boldsymbol{x}, \boldsymbol{u}_{\boldsymbol{k}}\right)$ is bijective, of class $\mathcal{C}^{1}$ and, for all $k \in \mathbb{N}^{*}$ and for all admissible input vector $\boldsymbol{u}_{\boldsymbol{k}}$, its Jacobian matrix is bounded on $\mathcal{E}\left(\hat{\boldsymbol{x}}_{\boldsymbol{k}}, \sigma_{k}^{2} P_{k}\right)$, the ellipsoid containing all presumed values of the true state vector $\boldsymbol{x}_{\boldsymbol{k}}^{*}$, where the center of the ellipsoid $\hat{\boldsymbol{x}}_{\boldsymbol{k}} \in \mathbb{R}^{n}$ is the estimate of $\boldsymbol{x}_{\boldsymbol{k}}^{*}, \sigma_{k}>0, P_{k} \succ 0_{n \times n}$.

Our aim in the sequel is to design an estimation algorithm for the system (1), that is a progression law for the ellipsoid $\mathcal{E}_{k}:=\mathcal{E}\left(\hat{\boldsymbol{x}}_{\boldsymbol{k}}, \sigma_{k}^{2} P_{k}\right)$, in light of the current measurements and noise constraints, such that

(i) a set that contains all possible values of the true state vector $\boldsymbol{x}_{\boldsymbol{k}}^{*}$ is quantified at each time step $k$;

(ii) the (a posteriori) output error vector $y_{k}-F_{k} \hat{\boldsymbol{x}}_{\boldsymbol{k}}$ is acceptable, i.e., it remains in the interior of the ellipsoid (2b) enclosing all possible values of the disturbance vectors $\boldsymbol{v}_{\boldsymbol{k}}$, i.e.

$$
\left(\boldsymbol{y}_{\boldsymbol{k}}-F_{k} \hat{\boldsymbol{x}}_{\boldsymbol{k}}\right)^{T} V_{k}^{-1}\left(\boldsymbol{y}_{\boldsymbol{k}}-F_{k} \hat{\boldsymbol{x}}_{\boldsymbol{k}}\right) \leq 1, \forall k \in \mathbb{N}^{*}
$$

(iii) the estimator is input-to-sate stable (ISS).

Since there is not any information about the measurement noise vector $\boldsymbol{v}_{\boldsymbol{k}}$, except the fact that $\left(\boldsymbol{v}_{\boldsymbol{k}}=\boldsymbol{y}_{\boldsymbol{k}}-F_{k} \boldsymbol{x}_{\boldsymbol{k}}^{*}\right) \in$ $\mathcal{E}\left(\mathbf{0}, V_{k}\right)$, the best state estimate is each one for which the output error is acceptable, that is, $\left(\boldsymbol{y}_{\boldsymbol{k}}-F_{k} \hat{\boldsymbol{x}}_{\boldsymbol{k}}\right) \in \mathcal{E}\left(\mathbf{0}, V_{k}\right)$.

It is assumed that the state vector $\boldsymbol{x}_{\boldsymbol{k}}^{*}$ belongs to a known ellipsoid $\mathcal{E}\left(\hat{\boldsymbol{x}}_{\boldsymbol{0}}, \sigma_{0}^{2} P_{0}\right)$, where $\hat{\boldsymbol{x}}_{\boldsymbol{0}}$ is the initial estimate of $\boldsymbol{x}_{\mathbf{0}}^{*}, P_{0} \succ 0$ and $\sigma_{0}>0$.

\section{TIME UPDATE}

At this stage, we compute, at each time step $k$, the ellipsoid $\mathcal{E}_{k / k-1}$ containing the "reach set" from $\mathcal{E}_{k-1}$ of the current state vector $\boldsymbol{x}_{\boldsymbol{k}}^{*}$, that evolves obeying to the plant dynamics described by (1a) and affected by the unknown noise vector $\boldsymbol{w}_{\boldsymbol{k}-1}: \mathcal{E}_{k / k-1} \supseteq\left\{\boldsymbol{z} \in \mathbb{R}^{n} \mid \boldsymbol{z}=\boldsymbol{\varphi}_{\boldsymbol{k}-\mathbf{1}}(\boldsymbol{x})+\boldsymbol{w}, \boldsymbol{x} \in \mathcal{E}_{k-1}, \boldsymbol{w} \in \mathcal{E}\left(\mathbf{0}, W_{k-1}\right)\right\}$. This is done by performing the vector sum of the ellipsoid $\mathcal{E}\left(\mathbf{0}, W_{k-1}\right)$ and a linear transformation of $\mathcal{E}_{k-1}$.

Lemma 1: Let $\boldsymbol{x}_{\boldsymbol{k}-\mathbf{1}}^{*} \in \mathcal{E}_{k-1}$ obeying to (1a) and (2a); if

$$
\begin{aligned}
\hat{\boldsymbol{x}}_{\boldsymbol{k} / \boldsymbol{k}-\mathbf{1}} & =\boldsymbol{\varphi}_{\boldsymbol{k}-\mathbf{1}}\left(\hat{\boldsymbol{x}}_{\boldsymbol{k}-\mathbf{1}}\right), \\
P_{k / k-1} & =\frac{1}{1-\rho}\left(\hat{\Phi}_{k-1}+\Delta_{k-1}\right)^{T} P_{k-1}\left(\hat{\Phi}_{k-1}+\Delta_{k-1}+\frac{1}{\rho \sigma_{k-1}^{2}} W_{k-1},\right. \\
\sigma_{k / k-1}^{2} & =\sigma_{k-1}^{2} ;
\end{aligned}
$$

where $\forall \boldsymbol{\xi} \in \mathbb{R}^{n}, \Phi_{k}(\boldsymbol{\xi}):=\Phi\left(\boldsymbol{\xi}, \boldsymbol{u}_{\boldsymbol{k}}\right)=\left.\frac{\partial \varphi\left(\boldsymbol{x}, \boldsymbol{u}_{\boldsymbol{k}}\right)}{\partial \boldsymbol{x}}\right|_{x=\xi}$ and $\hat{\Phi}_{k}:=\Phi_{k}\left(\hat{\boldsymbol{x}}_{\boldsymbol{k}}\right)$ and where $\Delta_{k} \in \mathbb{R}^{n \times n}$ is such that

$$
\Delta_{k}+\hat{\Phi}_{k}-\Phi_{k}(\boldsymbol{\xi}) \succeq 0_{n \times n}, \forall \boldsymbol{\xi} \in \mathcal{E}_{k-1}
$$

then

$$
\forall \rho \in] 0,1\left[, \boldsymbol{x}_{\boldsymbol{k}}^{*} \in \mathcal{E}\left(\hat{\boldsymbol{x}}_{k / k-1}, \sigma_{k / k-1}^{2} P_{k / k-1}\right)=: \mathcal{E}_{k / k-1}\right.
$$

Proof. $c f$. the appendix.

The optimal value of $\rho$ is the one that minimizes, at each step $k$, either the squared volume of the ellipsoid $\mathcal{E}_{k / k-1}$, i.e.,$\rho=\rho_{k}^{\star}=\arg \min \left(\sigma_{k-1}^{2 n} \operatorname{det} P_{k / k-1}\right)$ or the sum of the squared lengths of its axes, i.e., $\rho=\rho_{k}^{\diamond}=\arg \min \left(\sigma_{k-1} \operatorname{tr} P_{k / k-1}\right)$. These values and the methods of their obtention are given in details in [5].

\section{OBservation UPDATE}

The observation equation (1b) and the inequality (2b) define an other bounding set for the vector $\boldsymbol{x}_{\boldsymbol{k}}^{* 1}$. Indeed, it is clear that $\boldsymbol{x}_{\boldsymbol{k}}^{*} \in \mathcal{S}_{k}$, where $\mathcal{S}_{k}:=\left\{\boldsymbol{x} \in \mathbb{R}^{n} \mid\left(\boldsymbol{y}_{\boldsymbol{k}}-F_{k} \boldsymbol{x}\right)^{T} V_{k}^{-1}\left(\boldsymbol{y}_{\boldsymbol{k}}-F_{k} \boldsymbol{x}\right) \leq 1\right\}$. Thus, it remains to perform, at each iteration, the intersection between the ellipsoid $\mathcal{E}_{k / k-1}$ (obtained in the previous section) and the set $\mathcal{S}_{k}$. This intersection does not result, in general, in an ellipsoid and has to be circumscribed by an ellipsoid :

Lemma 2: If

$$
\begin{aligned}
\hat{\boldsymbol{x}}_{\boldsymbol{k}} & =\hat{\boldsymbol{x}}_{\boldsymbol{k} / \boldsymbol{k}-\mathbf{1}}+K_{k} \boldsymbol{\delta}_{\boldsymbol{k}}, \\
P_{k} & =\left(I_{n}-K_{k} F_{k}\right) P_{k / k-1}, \\
\sigma_{k}^{2} & =\sigma_{k / k-1}^{2}+\omega\left(1-\boldsymbol{\delta}_{k}^{T}\left(\omega F_{k} P_{k / k-1} F_{k}^{T}+V_{k}\right)^{-1} \boldsymbol{\delta}_{k}\right)
\end{aligned}
$$

where the gain matrix and the innovation are given by :

\footnotetext{
${ }^{1}$ The observation update stage of this algorithm is identical to that of the state estimation algorithm for linear systems [12].
} 


$$
\begin{aligned}
K_{k} & =\omega P_{k / k-1} F_{k}^{T}\left(\omega F_{k} P_{k / k-1} F_{k}^{T}+V_{k}\right)^{-1} \\
\boldsymbol{\delta}_{\boldsymbol{k}} & =\boldsymbol{y}_{\boldsymbol{k}}-F_{k} \hat{\boldsymbol{x}}_{\boldsymbol{k} / \boldsymbol{k}-\mathbf{1}}
\end{aligned}
$$

then $\forall \omega \in \mathbb{R}_{+},\left(\mathcal{E}_{k / k-1} \cap \mathcal{S}_{k}\right) \subseteq \mathcal{E}\left(\hat{\boldsymbol{x}}_{k}, \sigma_{k}^{2} P_{k}\right)=\mathcal{E}_{k}$.

Now, we are interested in the derivation of the "optimal" value of $\omega$ with respect to a criterion to be chosen. Contrary to some algorithms of the literature [5], [6] that minimize the size of the ellipsoid $\mathcal{E}\left(\hat{\boldsymbol{x}}_{\boldsymbol{k}}, \sigma_{k}^{2} P_{k}\right)$, the optimal value of $\omega$ chosen here is the one that guarantees the stability of the algorithm given by the equations (4) and (7).

Lemma 3: The solution of $\min _{\omega \in \mathbb{R}_{+}} \max _{v_{k} \in \mathcal{E}\left(0, V_{k}\right)} \mathscr{V}^{*}-\mathscr{V}_{-}^{*}$, where $\mathscr{V}^{*}{ }_{k}=\left(\boldsymbol{x}_{\boldsymbol{k}}^{*}-\hat{\boldsymbol{x}}_{\boldsymbol{k}}\right)^{T} P_{k}^{-1}\left(\boldsymbol{x}_{\boldsymbol{k}}^{*}-\hat{\boldsymbol{x}}_{\boldsymbol{k}}\right)$, is the solution of $\min _{\omega \in \mathbb{R}_{+}} \sigma_{k}^{2}$, which is $\omega_{k}^{*}=\left\{\begin{array}{ll}0 & \text { if }\left\|\boldsymbol{\delta}_{k}\right\|_{V_{k}^{-1}} \leq 1, \\ \varpi_{k} & \text { otherwise }\end{array}\right.$ where (the statements i and ii are equivalent) i. $\varpi_{k}$ is the unique real positive solution of the equation $\beta \sum_{i=1}^{p} \frac{\alpha_{i}^{2}}{\left(\gamma_{i} \omega+1\right)^{2}}=1$ in the unknown $\omega$, where $\alpha_{i}=\alpha_{k_{i}}=\frac{\boldsymbol{u}_{\boldsymbol{k}_{i}}{ }^{T} \bar{V}_{k} \boldsymbol{\delta}_{\boldsymbol{k}}}{\left\|\boldsymbol{\delta}_{\boldsymbol{k}}\right\|_{V_{k}{ }^{-1}}}, \beta=\beta_{k}=\left\|\boldsymbol{\delta}_{\boldsymbol{k}}\right\|_{V_{k}^{-1}}^{2}, \gamma_{i}=\gamma_{k_{i}} \in \mathbb{R}_{+}$, satisfying $\operatorname{det}\left(F_{k} P_{k / k-1} F_{k}^{T}-\gamma_{k_{i}} V_{k}\right)=0, \boldsymbol{u}_{\boldsymbol{k}_{\boldsymbol{i}}} \in \mathbb{R}^{p}$ are such that $\bar{V}_{k} F_{k} P_{k / k-1} F_{k}^{T} \bar{V}_{k}^{T} \boldsymbol{u}_{\boldsymbol{k}_{\boldsymbol{i}}}=\gamma_{i} \boldsymbol{u}_{\boldsymbol{k}_{\boldsymbol{i}}}(i \in\{1,2, \ldots, p\})$ and where $\bar{V}_{k}$ satisfies $\bar{V}_{k}^{T} \bar{V}_{k}=V_{k}^{-1}\left(e \cdot g . \bar{V}_{k}=V_{k}^{-\frac{1}{2}}\right)$. ii. $\varpi_{k}$ is the unique real positive eigenvalue of the matrix $\Xi_{k} \in \mathbb{R}^{2 p \times 2 p}$ :

$$
\Xi_{k}=\left(\begin{array}{ccccc}
0 & 1 & 0 & \cdots & 0 \\
0 & 0 & 1 & \cdots & 0 \\
\vdots & \vdots & \vdots & \ddots & \vdots \\
0 & 0 & 0 & \cdots & 1 \\
-\frac{\xi_{k_{0}}}{\xi_{k_{2 p}}} & -\frac{\xi_{k_{1}}}{\xi_{k_{2 p}}} & -\frac{\xi_{k_{2}}}{\xi_{k_{2 p}}} & \cdots & -\frac{\xi_{k_{p-1}}}{\xi_{k_{2 p}}}
\end{array}\right)
$$

where $\xi_{k_{i}}, i \in\{0, \ldots, 2 p\}$, are the components of the row-vector $\boldsymbol{\xi}_{\boldsymbol{k}}=\sum_{i=1}^{p} \alpha_{k_{i}}^{2} \boldsymbol{q}_{\boldsymbol{k}_{1}} *\left(\boldsymbol{q}_{\boldsymbol{k}_{2}} \cdots *\left(\boldsymbol{q}_{\boldsymbol{k}_{i-1}} *\left(\left(\boldsymbol{q}_{\boldsymbol{k}_{i}}-\boldsymbol{d}_{\boldsymbol{k}}\right)\right.\right.\right.$ $\left.\left.*\left(\boldsymbol{q}_{\boldsymbol{k}_{i+1}} \cdots *\left(\boldsymbol{q}_{\boldsymbol{k}_{p-1}} * \boldsymbol{q}_{\boldsymbol{k}_{p}}\right)\right)\right)\right), \boldsymbol{q}_{\boldsymbol{k}_{i}}=\left(\begin{array}{ll}\gamma_{k_{i}} & 1\end{array}\right) *\left(\begin{array}{ll}\gamma_{k_{i}} & 1\end{array}\right)=\left(\begin{array}{lll}\gamma_{k_{i}}^{2} & 2 \gamma_{k_{i}} & 1\end{array}\right), \boldsymbol{d}_{\boldsymbol{k}}=\left(\begin{array}{lll}0 & 0 & \beta_{k}\end{array}\right), *$ is the convolution operator ${ }^{2}$.

\section{Algorithm's PROPERTIES AND STABILITY ANALYSIS}

In this section, we show that the proposed algorithm with $\omega=\omega_{k}^{*}$ computed by the aid of Lemma 3 fulfills the expectations $\boldsymbol{i}-\boldsymbol{i i \boldsymbol { i }}$ expressed in the section I.

Definition 1 ([13]) The system $\boldsymbol{z}_{k}=f\left(\boldsymbol{z}_{k-1}, \boldsymbol{u}_{\boldsymbol{k - 1}}\right)$ is input-to-state stable (ISS) if there exist a $\mathscr{K} \mathscr{L}$-function ${ }^{3} \mu$ : $\mathbb{R}_{+} \times \mathbb{R}_{+} \longrightarrow \mathbb{R}_{+}$and a $\mathscr{K}$-function $\chi: \mathbb{R}_{+} \longrightarrow \mathbb{R}_{+}$such that, for each bounded input $\boldsymbol{u}_{\boldsymbol{k}}$, it holds that $\left\|\boldsymbol{z}_{k}\right\| \leq \mu\left(\left\|\boldsymbol{z}_{0}\right\|, k\right)+\chi(\|\boldsymbol{u}\|)$.

Theorem 1: Consider the state estimation algorithm (4) (where $\rho=\rho_{k-1}$ ) and (7) for the model (1a). The following propositions are true for all $k \in \mathbb{N}^{*}$ and for all $0<\rho<1$

$\boldsymbol{i}$. if $\boldsymbol{x}_{\mathbf{0}}^{*} \in \mathcal{E}_{0}$ then $\boldsymbol{x}_{\boldsymbol{k}}^{*} \in \mathcal{E}_{k}$ for all $\omega \in \mathbb{R}_{+}$;

Furthermore, if $\omega=\omega_{k}^{*}$, we have the following properties

ii. the sequence $\left(\sigma_{k}\right)_{k \in \mathbb{N}^{*}}$ - which represents an upper bound on the weighted norm of the estimation error vector $\left\|\boldsymbol{x}_{\boldsymbol{k}}^{*}-\hat{\boldsymbol{x}}_{\boldsymbol{k}}\right\|_{P_{k}^{-1}}$ - is decreasing and convergent on $\mathbb{R}_{+}$;

iii. the a posteriori output error vector is always acceptable, i.e., $\left\|\boldsymbol{y}_{\boldsymbol{k}}-F_{k} \hat{\boldsymbol{x}}_{\boldsymbol{k}}\right\|_{V_{k}^{-1}} \leq 1$.

Moreover, if there exists positive reals $a_{1}, a_{2}, b_{1}$ and $b_{2}$ such that the following inequalities hold for a finite $m \in \mathbb{N}^{*}$ and for all $k \in \mathbb{N}$ :

$$
\begin{aligned}
& a_{1} I_{n} \geq \sum_{i=k}^{k+m-1} \widetilde{\Phi}_{k+m, i+1} W_{i} \widetilde{\Phi}_{k+m, i+1}^{T} \geq a_{2} I_{n} \\
& b_{1} I_{n} \leq \sum_{i=k}^{k+s_{k}(m)} \widetilde{\Phi}_{i, k+s_{k}(m)}^{T} F_{i}^{T} V_{i} F_{i} \widetilde{\Phi}_{i, k+s_{k}(m)} \leq b_{2} I_{n}
\end{aligned}
$$

where

$$
\begin{aligned}
& \widetilde{\Phi}_{k+j, k}=\widetilde{\Phi}_{k+j, k+j-1} \widetilde{\Phi}_{k+j-1, k+j-2} \cdots \widetilde{\Phi}_{k+1, k}, \\
& \widetilde{\Phi}_{k+1, k}=\hat{\Phi}_{k}, \quad \widetilde{\Phi}_{k, k+j}=\widetilde{\Phi}_{k+j, k}^{-1}, \text { and } \widetilde{\Phi}_{k, k}=I_{n}
\end{aligned}
$$

${ }^{2}$ If $\boldsymbol{x}=\left(x_{r} \cdots x_{0}\right)$ and $\boldsymbol{y}=\left(y_{s} \cdots y_{0}\right)$, then (assuming that $\left.r \leq s\right) \boldsymbol{z}=\boldsymbol{x} * \boldsymbol{y}=\left(z_{r+s} z_{r+s-1} \cdots z_{0}\right)$ where $z_{j}=\sum_{i=0}^{j} x_{i} y_{j-i}$ if $j \leq \min (r, s)=r$ and $z_{j}=\sum_{i=0}^{r} x_{i} y_{j-i}$ otherwise

${ }^{3}$ a function $\chi \mathbb{R}_{+} \longrightarrow \mathbb{R}_{+}$: is a $\mathscr{K}$-function if it is continuous, strictly increasing and $\chi(0)=0$; a function $\mu: \mathbb{R}_{+} \times \mathbb{R}_{+} \longrightarrow \mathbb{R}_{+}$is a $\mathscr{K} \mathscr{L}$-function if, for each fixed $t_{0} \geq 0$, the function $\mu\left(s, t_{0}\right)$ is a $\mathscr{K}$-function, and for each fixed $s_{0} \geq 0$, the function $\mu\left(s_{0}, t\right)$ is decreasing and $\mu(s, t) \rightarrow 0$ as $t \rightarrow 1$. 
and $s_{k}(m)$ is such that ${ }^{4}$

$$
\operatorname{Card} \mathcal{N}\left(\omega_{k}^{*}, \omega_{k+1}^{*}, \ldots, \omega_{k+s_{k}(m)-1}^{*}\right)=m \quad \forall k, s \in \mathbb{N}^{*}, \mathcal{N}\left(\omega_{k}^{*}, \ldots, \omega_{k+s-1}^{*}\right):=\left\{i \in \mathbb{N}^{*} \mid k \leq i<k+s, \omega_{i}^{*}>0\right\}
$$

then

$\boldsymbol{i v}$. the sequences $\left(\omega_{k}^{*}\right)_{k \in \mathbb{N}^{*}},\left(\hat{\boldsymbol{x}}_{\boldsymbol{k}}-\hat{\boldsymbol{x}}_{\boldsymbol{k} / \boldsymbol{k}-\mathbf{1}}\right)_{k \in \mathbb{N}^{*}}$ and $\left(P_{k}-P_{k / k-1}\right)_{k \in \mathbb{N}^{*}}$ are convergent in $\mathbb{R}_{+}, \mathbb{R}^{n}$ and $\mathbb{R}^{n \times n}$ respectively, with $\lim _{k \longrightarrow \infty} \omega_{k}^{*}=0, \lim _{k \rightarrow \infty} \hat{\boldsymbol{x}}_{\boldsymbol{k}}-\hat{\boldsymbol{x}}_{\boldsymbol{k} / \boldsymbol{k}-\mathbf{1}}=\mathbf{0}$ and $\lim _{k \rightarrow \infty} P_{k}-P_{k / k-1}=0_{n \times n}$

$\boldsymbol{v}$. the innovation vector tends to the interior of the ellipsoid $\mathcal{E}\left(\mathbf{0}, V_{k}\right)$, i.e., $\forall \varepsilon>0, \exists k_{\infty} \in \mathbb{N}^{*}, \forall k>k_{\infty}, \boldsymbol{\delta}_{k}^{T} V_{k}^{-1} \boldsymbol{\delta}_{k}<1+\varepsilon$; $\boldsymbol{v i}$. the volume and all the axes' lengths of $\mathcal{E}\left(\hat{\boldsymbol{x}}_{\boldsymbol{k}}, \sigma_{k}^{2} P_{k}\right)$ are bounded for all $k \in \mathbb{N}^{*}$;

$\boldsymbol{v i \boldsymbol { i }}$. the estimation error dynamic system of state vector $\widetilde{\boldsymbol{x}}_{\boldsymbol{k}}=\boldsymbol{x}_{\boldsymbol{k}}^{*}-\hat{\boldsymbol{x}}_{\boldsymbol{k}}$ is ISS.

Remark 1: It is worth to mention that if $\boldsymbol{w}_{\boldsymbol{k}}=0$, the ISS-Lyapunov function becomes a decreasing Lyapunov function for the estimation error, and the presented algorithm (in which $P_{k / k-1}=\hat{\Phi}_{k}^{T} P_{k-1} \hat{\Phi}_{k}$ ) is an asymptotic state estimator for the system (1a) (with $\boldsymbol{w}_{\boldsymbol{k}}=0$ ) and this in spite of the presence of measurement noise $\boldsymbol{v}_{\boldsymbol{k}}$ and the nonlinearity.

\section{Application to an induction motor}

The presented algorithm is applied to a fifth-order two-phase nonlinear model of an induction motor which was already the subject of a large number of applications, especially in control designs (see [14]).

Using an Euler discretization of step size $h$ and adding the disturbance vectors $\boldsymbol{w}_{\boldsymbol{k}}$ and $\boldsymbol{v}_{\boldsymbol{k}}$, the complete discrete-time model in stator fixed $(a, b)$ reference frame is given by :

$$
\begin{aligned}
x_{1_{k+1}}^{*} & =x_{1_{k}}^{*}+h\left(-\mathrm{g} x_{1_{k}}^{*}+\frac{K}{T_{r}} x_{3_{k}}^{*}+K p x_{5_{k}}^{*} x_{4_{k}}^{*}+\frac{1}{s L_{s}} u_{1_{k}}\right)+w_{1 k} \\
x_{2_{k+1}}^{*} & =x_{2_{k}}^{*}+h\left(-\mathrm{g} x_{2_{k}}^{*}-K p x_{5_{k}}^{*} x_{3_{k}}^{*}+\frac{K}{T_{r}} x_{4_{k}}^{*}+\frac{1}{s L_{s}} u_{2_{k}}\right)+w_{2_{k}} \\
x_{3_{k+1}}^{*} & =x_{3_{k}}^{*}+h\left(\frac{M}{T_{r}} x_{1_{k}}^{*}-\frac{1}{T_{r}} x_{3_{k}}^{*}-p x_{5_{k}}^{*} x_{4_{k}}^{*}\right)+w_{3_{k}} \\
x_{4_{k+1}}^{*} & =x_{4_{k}}^{*}+h\left(\frac{M}{T_{r}} x_{2_{k}}^{*}+p x_{5_{k}}^{*} x_{3_{k}}^{*}-\frac{1}{T_{r}} x_{4_{k}}^{*}\right)+w_{4_{k}} \\
x_{5_{k+1}}^{*} & =x_{5_{k}}^{*}+h\left(\frac{p M}{J L_{r}}\left(x_{3_{k}}^{*} x_{2_{k}}^{*}-x_{4_{k}}^{*} x_{1_{k}}^{*}\right)-\frac{T_{L}}{J}\right)+w_{5_{k}} \\
y_{1_{k}} & =x_{1_{k}}^{*}+v_{1_{k}}, y_{2_{k}}=x_{2_{k}}^{*}+v_{2_{k}}
\end{aligned}
$$

Simulations are performed using the same numerical values as in [14]. The input signals are $: u_{1_{k}}=325 \cos (0.03 k)$ and $u_{2_{k}}=325 \sin (0.003 k)$. The noises vectors $\boldsymbol{w}_{\boldsymbol{k}-\boldsymbol{1}}$ and $\boldsymbol{v}_{\boldsymbol{k}}$ verify $(2)$, where $W_{k-1}=0.05^{2} \operatorname{diag}\left(x_{i \in\{1, \ldots, 5\}}^{2}\right)$ and $V_{k}=0.05^{2} \operatorname{diag}_{j \in\{1,2\}}\left(y_{j_{k}}^{2}\right)$. The parameter $\rho_{k}$ is chosen such that the trace of the matrix $\sigma_{k / k-1}^{2} P_{k / k-1}$ is minimized at each iteration; and the weighting matrix $\Delta_{k}$ is chosen as follows :

$$
\Delta_{k}= \begin{cases}\frac{10^{-3}\left(\left\|\boldsymbol{\delta}_{\boldsymbol{k}}\right\|_{V_{k}^{-1}}-1\right)}{\left\|\boldsymbol{\delta}_{\boldsymbol{k}}\right\|_{V_{k}^{-1}}} I_{5}, & \text { if }\left\|\boldsymbol{\delta}_{\boldsymbol{k}}\right\|_{V_{k}^{-1}}>1, \\ \Delta_{k-1}, & \text { otherwise. }\end{cases}
$$

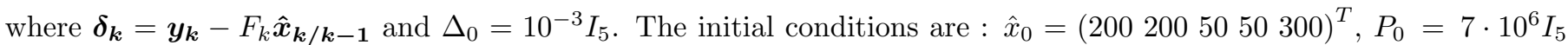
and $\sigma_{0}^{2}=0.1$ while the true initial state vector is : $x_{0}^{*}=10^{-3}\left(\begin{array}{lllll}1 & 1 & 1 & 1 & 1\end{array}\right)^{T}$. Because of important initial errors, and with

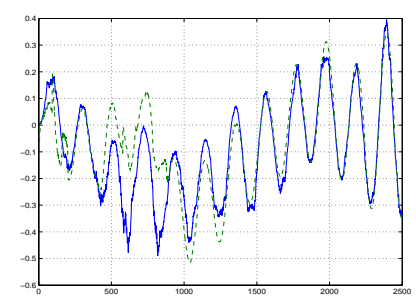

(a) $x_{3_{k}}^{*}(--), \hat{x}_{3_{k}}(-)$

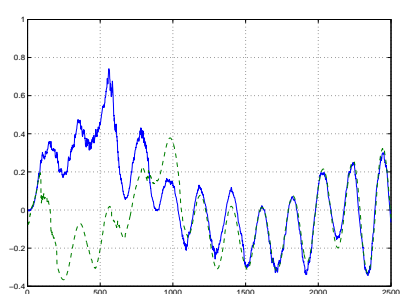

(b) $x_{4_{k}}^{*}(--), \hat{x}_{4_{k}}(-)$

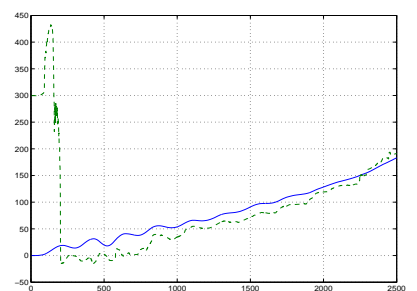

(c) $x_{5_{k}}^{*}(--), \hat{x}_{5_{k}}(-)$

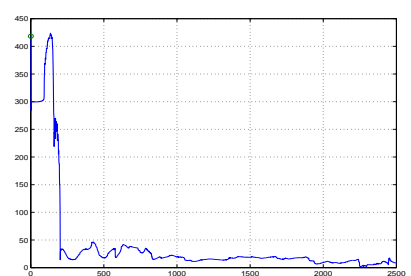

(d) $\left\|\boldsymbol{x}_{\boldsymbol{k}}^{*}-\hat{\boldsymbol{x}}_{\boldsymbol{k}}\right\|$

Fig. 1. Simulation results : estimated and true state components and error norm.

a view of precision, the first 5 samples were skipped from all the figures. This fact demonstrates that the estimation error decreases sharply soon after the first iterations.

\footnotetext{
${ }^{4}$ In the sequence $\left\{\omega_{k}^{*}, \omega_{k+1}^{*}, \ldots, \omega_{i}^{*}, \ldots, \omega_{k+s_{k}(m)-1}^{*}\right\}$ of length $s_{k}(m)$, there must be exactly $m$ times $\omega_{i}^{*} \neq 0\left(i . e .\left\|\boldsymbol{\delta}_{\boldsymbol{i}}\right\|_{V_{k}^{-1}}>1\right)$ and the rest $s_{k}(m)-m$ of $\omega_{i}^{*}$ are zero.

Card $S$ is the cardinal of the set $S=\left\{s_{1}, \ldots, s_{m}\right\}$ and is equal to the finite number $m$ of its elements.
} 


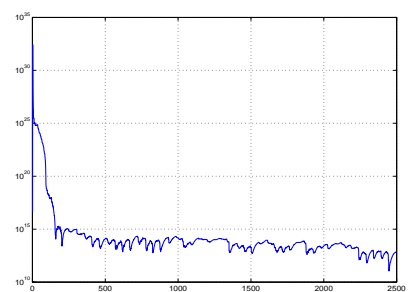

(a) $\sqrt{\operatorname{det}\left(\sigma_{k}^{2} P_{k}\right)}$

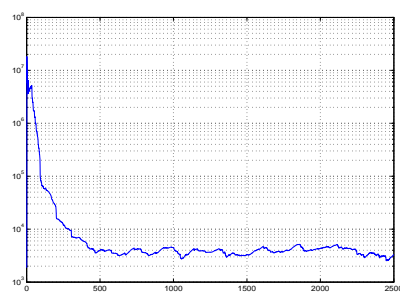

(b) $\sqrt{\operatorname{tr}\left(\sigma_{k}^{2} P_{k}\right)}$

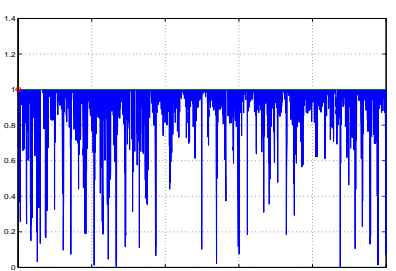

(c) $\left\|\boldsymbol{y}_{\boldsymbol{k}}-H_{k} \hat{\boldsymbol{x}}_{\boldsymbol{k}}\right\|_{V_{k}^{-1}}^{2}$

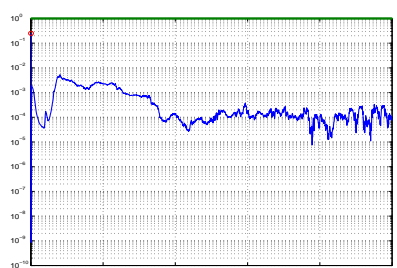

(d) $\left\|\boldsymbol{x}_{\boldsymbol{k}}^{*}-\hat{\boldsymbol{x}}_{\boldsymbol{k}}\right\|_{\left(\sigma_{k}^{2} P_{k}\right)^{-1}}^{2}$

Fig. 2. Simulation results : measures of $\mathcal{E}\left(\hat{\boldsymbol{x}}_{\boldsymbol{k}}, \sigma_{k}^{2} P_{k}\right)$ and weighted output and estimation error norms.

The figures show the satisfying performances of the proposed observer to track the true state with unknown bounded noises, without the need for rotor speed measurement and even with bad initialisations. Indeed,

- the assertion $\left(\boldsymbol{x}_{\boldsymbol{k}}^{*}-\hat{\boldsymbol{x}}_{\boldsymbol{k}}\right)^{T}\left(\sigma_{k}^{2} P_{k}\right)^{-1}\left(\boldsymbol{x}_{\boldsymbol{k}}^{*}-\hat{\boldsymbol{x}}_{\boldsymbol{k}}\right)<1$ shown in the figure $2(\mathrm{~d})$ guarantees that the statement $\boldsymbol{x}_{\boldsymbol{k}}^{*} \in \mathcal{E}\left(\hat{\boldsymbol{x}}_{\boldsymbol{k}}, \sigma_{k}^{2} P_{k}\right)$ is always verified : the aim $\boldsymbol{i}$ of Section I is fulfilled;

- the (a posteriori) output error is always in the interior of the noise ellipsoid $\mathcal{E}\left(\mathbf{0}, V_{k}\right)$ : the aim $\boldsymbol{i i}$ is fulfilled (cf. fig. $2(\mathrm{c}))$;

- the estimation error norm is significantly decreasing in the first samplings and remains small during all the simulation horizon;

- the volume and the sum of the squared axes' lengths are roughly decreasing;

- the observation updating is performed in $53 \%$ of samples, i.e., among the 2500 samples of the simulation, $\omega_{k}=0$ for 1173 of them; this is why this algorithm is fast.

\section{Conclusion}

A recursive state bounding technique for systems with nonlinear dynamics has been presented. An ellipsoid that encloses all the possible values of the state vector and which is compatible with the bounds of the noises and the linearization errors was determined at each sampling time. As the Kalman filter, the algorithm has been decomposed into time update and observation update steps. During the time update stage, an ellipsoid that encloses the vector sum of two ellipsoids, one containing the "predicted" state vector $\hat{\boldsymbol{x}}_{\boldsymbol{k} / \boldsymbol{k}-\boldsymbol{1}}$ of the previous sampling time and the other, the process noises. During the observation update step the set of state vector compatible with the current measure and the noise bound is isolated. The observation update stage may seem to entail heavy computations, but these operations are skipped as soon as the a priori output error is acceptable, that is, when $\left(\boldsymbol{y}_{\boldsymbol{k}}-F_{k} \hat{\boldsymbol{x}}_{\boldsymbol{k} / \boldsymbol{k}-\mathbf{1}}\right) \in \mathcal{E}\left(\mathbf{0}, V_{k}\right)$, which happens often and which makes the algorithm faster.

\section{APPENDIX}

\section{ProOFs}

Proof of the lemma 1. First, the following proposition is proved :

Proposition 1: There exists a bounded matrix $\Delta_{k} \in \mathbb{R}^{n \times n}$ satisfying (5) such that for all $\boldsymbol{x} \in \mathcal{E}_{k}$, $\boldsymbol{\varphi}_{\boldsymbol{k}}(\boldsymbol{x}) \in \mathcal{E}\left(\boldsymbol{\varphi}_{\boldsymbol{k}}\left(\hat{\boldsymbol{x}}_{\boldsymbol{k}}\right), \sigma_{k}^{2}\left(\hat{\Phi}_{k}+\Delta_{k}\right) P_{k}\left(\hat{\Phi}_{k}+\Delta_{k}\right)^{T}\right)$.

The existence of such a bounded matrix $\Delta_{k}$ is guaranteed by the boundedness of the ellipsoid $\mathcal{E}_{k}$ (and thus of $P_{k}$ ) which will be proved later.

Let $\phi:[0,1] \longrightarrow \mathbb{R}^{n}, \tau \longmapsto \phi(\tau)=\boldsymbol{\varphi}_{\boldsymbol{k}}\left(\hat{\boldsymbol{x}}_{\boldsymbol{k}}+\tau\left(\boldsymbol{x}-\hat{\boldsymbol{x}}_{\boldsymbol{k}}\right)\right)$; when $\tau \in[0,1], \hat{\boldsymbol{x}}_{\boldsymbol{k}}+\tau\left(\boldsymbol{x}-\hat{\boldsymbol{x}}_{\boldsymbol{k}}\right) \in\left[\boldsymbol{x}, \hat{\boldsymbol{x}}_{\boldsymbol{k}}\right]^{5}$. its derivative : $\dot{\phi}(\tau)=\Phi_{k}\left(\hat{\boldsymbol{x}}_{\boldsymbol{k}}+\tau\left(\boldsymbol{x}-\hat{\boldsymbol{x}}_{\boldsymbol{k}}\right)\right) \cdot\left(\boldsymbol{x}-\hat{\boldsymbol{x}}_{\boldsymbol{k}}\right)$.

$\exists \theta \in] 0,1\left[, \phi(1)-\phi(0)=\int_{0}^{1} \dot{\phi}(\tau) \mathrm{d} \tau=\dot{\phi}(\theta)\right.$, so for this $\theta, \boldsymbol{\varphi}_{\boldsymbol{k}}(\boldsymbol{x})=\boldsymbol{\varphi}_{\boldsymbol{k}}\left(\hat{\boldsymbol{x}}_{\boldsymbol{k}}\right)+\Phi_{k}\left(\hat{\boldsymbol{x}}_{\boldsymbol{k}}+\theta\left(\boldsymbol{x}-\hat{\boldsymbol{x}}_{\boldsymbol{k}}\right)\right)\left(\boldsymbol{x}-\hat{\boldsymbol{x}}_{\boldsymbol{k}}\right)$. Let $\boldsymbol{\xi}:=\hat{\boldsymbol{x}}_{\boldsymbol{k}}+\theta\left(\boldsymbol{x}-\hat{\boldsymbol{x}}_{\boldsymbol{k}}\right)$, the latest can be rewritten as :

$$
\exists \boldsymbol{\xi} \in] \boldsymbol{x}, \hat{\boldsymbol{x}}_{\boldsymbol{k}}\left[, \boldsymbol{\varphi}_{\boldsymbol{k}}(\boldsymbol{x})=\boldsymbol{\varphi}_{\boldsymbol{k}}\left(\hat{\boldsymbol{x}}_{\boldsymbol{k}}\right)+\Phi_{k}(\boldsymbol{\xi})\left(\boldsymbol{x}-\hat{\boldsymbol{x}}_{\boldsymbol{k}}\right)\right.
$$

Since $\hat{\boldsymbol{x}}_{\boldsymbol{k}} \in \mathcal{E}_{k}$ and an ellipsoid is a convex set,

$$
\left.\boldsymbol{x} \in \mathcal{E}_{k} \Rightarrow\right] \boldsymbol{x}, \hat{\boldsymbol{x}}_{\boldsymbol{k}}\left[\subset \mathcal{E}_{k} \Rightarrow \boldsymbol{\xi} \in \mathcal{E}_{k} .\right.
$$

As $\varphi_{\boldsymbol{k}}$ is bijective, $\Phi_{k}(\boldsymbol{\xi})$ is invertible; then, using (14), it comes that

$$
\begin{array}{r}
\boldsymbol{x} \in \mathcal{E}_{k} \Leftrightarrow\left(\boldsymbol{x}-\hat{\boldsymbol{x}}_{\boldsymbol{k}}\right)^{T} \Phi_{k}(\boldsymbol{\xi})^{T}\left(\Phi_{k}(\boldsymbol{\xi})^{T} P_{k} \Phi_{k}(\boldsymbol{\xi})\right)^{-1} \Phi_{k}(\boldsymbol{\xi})\left(\boldsymbol{x}-\hat{\boldsymbol{x}}_{\boldsymbol{k}}\right) \leq \sigma_{k}^{2} \\
\Leftrightarrow\left(\boldsymbol{\varphi}_{\boldsymbol{k}}(\boldsymbol{x})-\boldsymbol{\varphi}_{\boldsymbol{k}}\left(\hat{\boldsymbol{x}}_{\boldsymbol{k}}\right)\right)^{T}\left(\hat{\Phi}_{k}(\boldsymbol{\xi})^{T} P_{k} \hat{\Phi}_{k}(\boldsymbol{\xi})\right)^{-1}\left(\boldsymbol{\varphi}_{\boldsymbol{k}}(\boldsymbol{x})-\boldsymbol{\varphi}_{\boldsymbol{k}}\left(\hat{\boldsymbol{x}}_{\boldsymbol{k}}\right)\right) \leq \sigma_{k}^{2} .
\end{array}
$$

${ }^{5}$ a vector interval $[\boldsymbol{a}, \boldsymbol{b}]$ is defined as follows $[\boldsymbol{a}, \boldsymbol{b}]:=\left\{\boldsymbol{c} \in \mathbb{R}^{n} \mid \boldsymbol{c}=(1-\theta) \boldsymbol{a}+\theta \boldsymbol{b}, \theta \in[0,1]\right\}$ 
The inequality (5) means that $\forall \boldsymbol{\xi} \in \mathcal{E}_{k},\left(\left(\Delta_{k}+\hat{\Phi}_{k}\right)^{T} P_{k}\left(\Delta_{k}+\hat{\Phi}_{k}\right)\right)^{-1} \preceq\left(\Phi_{k}(\boldsymbol{\xi})^{T} P_{k} \Phi_{k}(\boldsymbol{\xi})\right)^{-1}$; thus (15), (16) and the latest inequality lead to

$$
\boldsymbol{x} \in \mathcal{E}_{k} \Rightarrow\left(\boldsymbol{\varphi}_{\boldsymbol{k}}(\boldsymbol{x})-\boldsymbol{\varphi}_{\boldsymbol{k}}\left(\hat{\boldsymbol{x}}_{\boldsymbol{k}}\right)\right)^{T}\left(\left(\hat{\Phi}_{k}+\Delta_{k}\right)^{T} P_{k}\left(\hat{\Phi}_{k}+\Delta_{k}\right)\right)^{-1}\left(\boldsymbol{\varphi}_{\boldsymbol{k}}(\boldsymbol{x})-\boldsymbol{\varphi}_{\boldsymbol{k}}\left(\hat{\boldsymbol{x}}_{\boldsymbol{k}}\right)\right) \leq \sigma_{k}^{2},
$$

this proves the Proposition 1. Now, it only remains to determine the ellipsoid that contains the sum of two ellipsoids :

Proposition 2 ([15]) Let $P_{1}, P_{2} \in \mathbb{R}^{s \times s} P_{1}, P_{2} \succ 0$ and let $\boldsymbol{c}_{1}, \boldsymbol{c}_{2} \in \mathbb{R}^{s}$ be two vectors; let

$\mathcal{E}\left(\boldsymbol{c}_{1}, P_{1}\right) \oplus \mathcal{E}\left(\boldsymbol{c}_{2}, P_{2}\right):=\left\{\boldsymbol{x} \in \mathbb{R}^{s} \mid \boldsymbol{x}=\boldsymbol{x}_{1}+\boldsymbol{x}_{2}, \boldsymbol{x}_{1} \in \mathcal{E}\left(\boldsymbol{c}_{1}, P_{1}\right), \boldsymbol{x}_{2} \in \mathcal{E}\left(\boldsymbol{c}_{2}, P_{2}\right)\right\}$ and let $\boldsymbol{c}^{*}=\boldsymbol{c}_{1}+\boldsymbol{c}_{2}$ and $P^{*}=\frac{1}{\mu} P_{1}+\frac{1}{1-\mu} P_{2}$ (for any given $0<\mu<1)$; then $\forall \mu \in] 0,1\left[, \mathcal{E}\left(\boldsymbol{c}_{1}, P_{1}\right) \oplus \mathcal{E}\left(\boldsymbol{c}_{2}, P_{2}\right) \subseteq \mathcal{E}\left(\boldsymbol{c}^{*}, P^{*}\right)\right.$.

This proposition is applied to the ellipsoids $(17)$ and $\mathcal{E}\left(\mathbf{0}, W_{k}\right)$, it can be deduced that for all

$\rho \in] 0,1\left[,\left(\boldsymbol{\varphi}_{\boldsymbol{k}}(\boldsymbol{x})-\boldsymbol{\varphi}_{\boldsymbol{k}}\left(\hat{\boldsymbol{x}}_{\boldsymbol{k}}\right)+\boldsymbol{w}_{\boldsymbol{k}}\right)^{T}\left(\frac{\sigma_{k}^{2}}{1-\rho}\left(\hat{\Phi}_{k}+\Delta_{k}\right)^{T} P_{k}\left(\hat{\Phi}_{k}+\Delta_{k}\right)+\frac{1}{\rho} W_{k}\right)\left(\boldsymbol{\varphi}_{\boldsymbol{k}}(\boldsymbol{x})-\boldsymbol{\varphi}_{\boldsymbol{k}}\left(\hat{\boldsymbol{x}}_{\boldsymbol{k}}\right)+\boldsymbol{w}_{\boldsymbol{k}}\right) \leq 1\right.$ and the Lemma 1 is proved.

Proof of the lemma 2. Using (7b) and (7d) and after some linear manipulations, we obtain :

$$
\begin{aligned}
K_{k} & =\omega P_{k} F_{k}^{T} V_{k}^{-1} \\
P_{k}^{-1} & =P_{k / k-1}^{-1}+\omega F_{k}^{T} V_{k}^{-1} F_{k}
\end{aligned}
$$

(19) shows that $P_{k} \succ 0_{n \times n}$ (since $P_{k / k-1} \succ 0_{n \times n}$ ). Substituting (18) in (7a) yields $\hat{\boldsymbol{x}}_{\boldsymbol{k}}=\hat{\boldsymbol{x}}_{\boldsymbol{k} / \boldsymbol{k}-\boldsymbol{1}}+\omega P_{k} F_{k}^{T} V_{k}^{-1} \boldsymbol{\delta}_{\boldsymbol{k}}$. Letting $\mathscr{V}_{k}:=\left(\boldsymbol{x}-\hat{\boldsymbol{x}}_{\boldsymbol{k}}\right)^{T} P_{k}^{-1}\left(\boldsymbol{x}-\hat{\boldsymbol{x}}_{\boldsymbol{k}}\right)$ and $\mathscr{V}_{k / k-1}:=\left(\boldsymbol{x}-\hat{\boldsymbol{x}}_{\boldsymbol{k} / \boldsymbol{k}-\mathbf{1}}\right)^{T} P_{k / k-1}^{-1}\left(\boldsymbol{x}-\hat{\boldsymbol{x}}_{\boldsymbol{k} / \boldsymbol{k}-\mathbf{1}}\right)$, the use of $(7 \mathrm{a}),(7 \mathrm{~b}),(19)$ and (7d) and some routine algebra leads to

$$
\mathscr{V}_{k}=\mathscr{V}_{k / k-1}-\omega \boldsymbol{\delta}_{\boldsymbol{k}}^{T}\left(\omega F_{k} P_{k / k-1} F_{k}^{T}+V_{k}\right)^{-1} \boldsymbol{\delta}_{\boldsymbol{k}}+\omega\left(\boldsymbol{y}_{\boldsymbol{k}}-F_{k} \boldsymbol{x}\right)^{T} V_{k}^{-1}\left(\boldsymbol{y}_{\boldsymbol{k}}-F_{k} \boldsymbol{x}\right), \forall \boldsymbol{x} \in \mathbb{R}^{n} .
$$

By the aid of (7c) and (20), we can see that, for any $\omega \in \mathbb{R}_{+}$, on the one hand $\boldsymbol{x} \in \mathcal{E}_{k} \Leftrightarrow \mathscr{V}_{k / k-1}+\omega\left(\boldsymbol{y}_{\boldsymbol{k}}-F_{k} \boldsymbol{x}\right)^{T} V_{k}^{-1}\left(\boldsymbol{y}_{\boldsymbol{k}}-F_{k} \boldsymbol{x}\right) \leq \sigma_{k / k-1}^{2}+\omega$, On the other hand, it is obvious that $\boldsymbol{x} \in\left(\mathcal{E}_{k / k-1} \cap \mathcal{S}_{k}\right) \Rightarrow \mathscr{V}_{k / k-1}+\omega\left(\boldsymbol{y}_{\boldsymbol{k}}-F_{k} \boldsymbol{x}\right)^{T} V_{k}^{-1}\left(\boldsymbol{y}_{\boldsymbol{k}}-F_{k} \boldsymbol{x}\right) \leq \sigma_{k / k-1}^{2}+\omega$, thus $\forall \boldsymbol{x} \in \mathbb{R}, \boldsymbol{x} \in\left(\mathcal{E}_{k / k-1} \cap \mathcal{S}_{k}\right) \Rightarrow \boldsymbol{x} \in \mathcal{E}_{k}$.

Proof of the lemma 3. To prove the first point of this lemma we need first to state the following proposition :

Proposition 3: The function $\mathscr{F} \boldsymbol{\alpha}, \beta, \gamma: \mathbb{R}_{+} \longrightarrow \mathbb{R}, \mathscr{F} \boldsymbol{\alpha}, \beta, \gamma(\omega)=\omega-\omega \beta \sum_{=} \frac{\alpha}{\gamma \omega+}$ has a global minimum on $\mathbb{R}_{+}$ for $\omega=\omega^{*}$ such that $\omega^{*}=\left\{\begin{array}{ll}\varpi & \text { if } \beta>1, \\ 0 & \text { otherwise; }\end{array}\right.$ where $\boldsymbol{\alpha}=\left(\alpha_{1} \alpha_{2} \cdots \alpha_{p}\right), \alpha_{i} \in \mathbb{R}$ such that $\sum_{i=1}^{p} \alpha_{i}^{2}=1 ; \beta \in \mathbb{R}_{+}$; $\boldsymbol{\gamma}=\left(\gamma_{1} \gamma_{2} \cdots \gamma_{p}\right), \gamma_{i} \in \mathbb{R}_{+}^{*}$; and $\varpi$ is the unique real positive solution of the equation $\beta \sum_{i=1}^{p} \frac{\alpha_{i}^{2}}{\left(\gamma_{i} \omega+1\right)^{2}}=1$.

Proof. (i) $\dot{\mathscr{F}} \boldsymbol{\alpha}, \beta, \gamma(\omega)=1-\beta \sum_{i=1}^{p} \frac{\alpha_{i}^{2}}{\left(\gamma_{i} \omega+1\right)^{2}}$ is strictly increasing on $\mathbb{R}_{+}$. (ii) $\sum_{i=1}^{p} \frac{\alpha_{i}^{2}}{\left(\gamma_{i} \omega+1\right)^{2}}<1$ for all $\omega>0$.

(iii) From (i) and (ii), it comes that the equation $\dot{\mathscr{F}} \boldsymbol{\alpha}, \beta, \gamma(\omega)=0$ has one and only one real strictly positive solution if and only if $\beta>1$; and if $0<\beta \leq 1, \dot{\mathscr{F}} \boldsymbol{\alpha}, \beta, \gamma(\omega)>0$ which means that $\mathscr{F} \boldsymbol{\alpha}, \beta, \boldsymbol{\gamma}$ is increasing.

(iv) $\frac{\mathrm{d}^{2}}{\mathrm{~d} \omega^{2}} \mathscr{F} \boldsymbol{\alpha}, \beta, \gamma\left(\omega^{*}\right)=\beta \sum_{=} \frac{\gamma \alpha}{(\gamma \omega+)}>, \forall \omega \in \mathbb{R}_{+}^{*}$. Finally, from (iii) and (iv), $\omega^{*}=\arg \left(\min _{\omega \in \mathbb{R}_{+}} \mathscr{F} \boldsymbol{\alpha}, \beta, \gamma(\omega)\right)$ and the result follows : $\beta \sum_{i=1}^{p} \frac{\alpha_{i}^{2}}{\left(\gamma_{i} \omega^{*}+1\right)^{2}}=1$, if $\beta>1, \omega^{*}=\min _{\omega \geq 0} \omega \Leftrightarrow \omega^{*}=0$, otherwise.

Proof of the Lemma 3. Using (20) and (1b), we can write

$$
\mathscr{V}^{*}{ }_{k}=\mathscr{V}^{*}{ }_{k / k-1}-\omega \boldsymbol{\delta}_{\boldsymbol{k}}{ }^{T}\left(\omega F_{k} P_{k / k-1} F_{k}^{T}+V_{k}\right)^{-1} \boldsymbol{\delta}_{\boldsymbol{k}}+\omega \boldsymbol{v}_{\boldsymbol{k}}{ }^{T} V_{k}^{-1} \boldsymbol{v}_{\boldsymbol{k}} .
$$

Let $X_{k}=\bar{V}_{k} F_{k} P_{k-1} F_{k}^{T} \bar{V}_{k}^{T}$ and $\overline{\boldsymbol{\delta}}_{\boldsymbol{k}}=\bar{V}_{k} \boldsymbol{\delta}_{\boldsymbol{k}}$. By the aid of (2b) and (21) it holds that

$$
\max _{v_{k} \in \mathcal{E}\left(\mathbf{0}, V_{k}\right)} \mathscr{V}_{k}^{*}=\mathscr{V}_{k / k-1}^{*}-\omega \overline{\boldsymbol{\delta}}_{k}^{T}\left(\omega X_{k}+I_{p}\right)^{-1} \overline{\boldsymbol{\delta}}_{k}+\omega .
$$

From (7c), we have

$$
\sigma_{k}^{2}-\sigma_{k-1}^{2}=\omega-\omega \overline{\boldsymbol{\delta}}_{k}^{T}\left(\omega X_{k}+I_{p}\right)^{-1} \overline{\boldsymbol{\delta}}_{\boldsymbol{k}}
$$


Thus (22) and (23) lead to

$$
\arg \min _{\omega>0} \max _{v_{k} \in \mathcal{E}\left(\mathbf{0}, V_{k}\right)}\left(\mathscr{V}^{*}-\mathscr{V}_{-}^{*}\right)=\arg \min _{\omega>0} \sigma_{k}^{2}
$$

As $X_{k}$ is symmetric, there exists $U_{k}=\left(\boldsymbol{u}_{\boldsymbol{k}_{\mathbf{1}}} \boldsymbol{u}_{\boldsymbol{k}_{\mathbf{2}}} \ldots \boldsymbol{u}_{\boldsymbol{k}_{\boldsymbol{p}}}\right) \in \mathbb{R}^{p \times p}$ such that $U_{k} U_{k}^{T}=I_{p}, U_{k}^{T} X_{k} U_{k}$ is diagonal and $X_{k} \boldsymbol{u}_{\boldsymbol{k}_{\boldsymbol{i}}}=\gamma_{k_{i}} \boldsymbol{u}_{\boldsymbol{k}_{\boldsymbol{i}}}$ for all $i \in\{1, \ldots, p\}$, where $\gamma_{k_{i}}$ and $\boldsymbol{u}_{\boldsymbol{k}_{\boldsymbol{i}}}$ are the $i^{\text {th }}$ eigenvalue and the associated eigenvector of $X_{k}$. It is obvious that $\boldsymbol{u}_{\boldsymbol{k}_{\boldsymbol{i}}}{ }^{T}\left(\omega_{k}^{*} X_{k}+I_{p}\right)^{-1} \boldsymbol{u}_{\boldsymbol{k}_{\boldsymbol{i}}}=\frac{1}{\left(\omega_{k}^{*} \gamma_{k_{i}}+1\right)}$, thus (23) becomes

$$
\begin{aligned}
\sigma_{k}^{2}-\sigma_{k-1}^{2} & =\omega-\omega \overline{\boldsymbol{\delta}}_{\boldsymbol{k}}^{T} U_{k} U_{k}^{T}\left(\omega X_{k}+I_{p}\right)^{-1} U_{k} U_{k}^{T} \overline{\boldsymbol{\delta}}_{\boldsymbol{k}} \\
& =\sigma_{k-1}^{2}+\omega\left(1-\sum_{i=1}^{p} \frac{\left(\boldsymbol{u}_{\boldsymbol{k}_{\boldsymbol{i}}}{ }^{T} \overline{\boldsymbol{\delta}}_{\boldsymbol{k}}\right)^{2}}{\omega \gamma_{k_{i}}+1}\right) \\
& =\omega\left(1-\beta_{k} \sum_{i=1}^{p} \frac{\alpha_{k_{i}}^{2}}{\omega \gamma_{k_{i}}+1}\right) .
\end{aligned}
$$

(i) as $P_{k-1} \succ 0_{n \times n}$, as $\bar{V}_{k}$ is non singular and as $F_{k}$ is a full row rank matrix, $X_{k} \succ 0_{p \times p}$ so $\gamma_{k_{i}}>0, \forall i \in\{1, \ldots, p\}$; (ii) as $\sum_{i=1}^{p}\left(\boldsymbol{u}_{\boldsymbol{k}_{\boldsymbol{i}}}{ }^{T} \overline{\boldsymbol{\delta}}_{\boldsymbol{k}}\right)^{2}=\overline{\boldsymbol{\delta}}_{\boldsymbol{k}}{ }^{T} U_{k} U_{k}^{T} \overline{\boldsymbol{\delta}}_{\boldsymbol{k}}=\left\|\overline{\boldsymbol{\delta}}_{\boldsymbol{k}}\right\|^{2}$, we have $\sum_{i=1}^{p} \alpha_{k_{i}}^{2}=\frac{\sum_{i=1}^{p}\left(\boldsymbol{u}_{\boldsymbol{k}_{\boldsymbol{i}}}{ }^{T} \overline{\boldsymbol{\delta}}_{\boldsymbol{k}}\right)^{2}}{\left\|\overline{\boldsymbol{\delta}}_{\boldsymbol{k}}\right\|^{2}}=1 ;(\mathrm{iii}) \beta_{k}=\left\|\overline{\boldsymbol{\delta}}_{\boldsymbol{k}}\right\|^{2} \geq 0$. Consequently, the function $\mathscr{F} \boldsymbol{\alpha}, \beta, \gamma(c f$. Proposition 3$)$ can be used to rewrite $(25)$ as $\sigma_{k}^{2}=\sigma_{k-1}^{2}+\mathscr{F} \boldsymbol{\alpha}, \beta, \boldsymbol{\gamma}(\omega)$; and it is clear that

$$
\omega_{k}^{*}=\arg \left(\min _{\omega \geq 0} \sigma_{k}^{2}\right)=\arg \left(\min _{\omega \geq 0} \mathscr{F} \boldsymbol{\alpha}, \beta, \gamma(\omega)\right) .
$$

Finally the Proposition 3 is used to deduce the value of $\omega_{k}^{*}$ and the point i is proved.

Now, the proof of the point ii is straightforward by considering the previous point and the fact that the roots of a polynomial are the eigenvalues of its companion matrix.

Proof of the theorem 1 .

$\boldsymbol{i}$. Let $\boldsymbol{x}_{\boldsymbol{k}-\mathbf{1}}^{*} \in \mathcal{E}\left(\hat{\boldsymbol{x}}_{\boldsymbol{k}-\mathbf{1}}, \sigma_{k-1}^{2} P_{k-1}\right)$. The use of the Lemma 1 gives

$\left(\boldsymbol{x}_{\boldsymbol{k}-\mathbf{1}}^{*} \in \mathcal{E}_{k-1} \Leftrightarrow \mathscr{V}^{*}{ }_{k-1} \leq \sigma_{k-1}^{2}\right) \Rightarrow\left(\boldsymbol{x}_{\boldsymbol{k}}^{*} \in \mathcal{E}_{k / k-1} \Leftrightarrow \mathscr{V}^{*}{ }_{k / k-1} \leq \sigma_{k / k-1}^{2}\right)$;

recalling that $X_{k}=\bar{V}_{k} F_{k} P_{k-1} F_{k}^{T} \bar{V}_{k}^{T}, \bar{\delta}_{k}=\bar{V}_{k} \boldsymbol{\delta}_{k}$ and $\bar{V}_{k}^{T} \bar{V}_{k}=V_{k}^{-1},(22)$ with (7c) yield to

$\mathscr{V}^{*}{ }_{k} \leq \sigma_{k / k-1}^{2}-\omega \overline{\boldsymbol{\delta}}_{\boldsymbol{k}}^{T}\left(\omega X_{k}+I_{p}\right)^{-1} \overline{\boldsymbol{\delta}}_{\boldsymbol{k}}+\omega=\sigma_{k}^{2} \Leftrightarrow \boldsymbol{x}_{\boldsymbol{k}}^{*} \in \mathcal{E}_{k}$.

Consequently $\left(\boldsymbol{x}_{\mathbf{0}}^{*} \in \mathcal{E}_{0} \Leftrightarrow \mathscr{V}^{*}{ }_{0} \leq \sigma_{0}^{2}\right) \Rightarrow\left(\mathscr{V}^{*}{ }_{1} \leq \sigma_{1}^{2} \Leftrightarrow \boldsymbol{x}_{\boldsymbol{k}}^{*} \in \mathcal{E}_{1}\right) \ldots \Rightarrow\left(\boldsymbol{x}_{\boldsymbol{k}-\mathbf{1}}^{*} \in \mathcal{E}_{k-1}\right) \Rightarrow\left(\boldsymbol{x}_{\boldsymbol{k}}^{*} \in \mathcal{E}_{k}\right)$.

$\boldsymbol{i i}$. Consider $\sigma_{k}^{2}$ defined in $(7 \mathrm{c})$ and $(4 \mathrm{c})$ with $\omega=\omega_{k}^{*}$ given by the lemma 3 . $\sigma_{k}^{2}=\sigma_{k-1}^{2}$, if $\left\|\boldsymbol{\delta}_{\boldsymbol{k}}\right\|_{V_{k}^{-1}} \leq 1$ and $\sigma_{k}^{2}=\sigma_{k-1}^{2}+\varpi_{k}\left(1-\beta_{k} \sum_{i=1}^{p} \frac{\alpha_{k_{i}}^{2}}{\varpi_{k} \gamma_{k_{i}}+1}\right)$, otherwise; where $\alpha_{k_{i}}, \beta_{k}, \gamma_{k_{i}}$ and $\varpi_{k}$ are defined in Lemma 3. As $\gamma_{k_{i}} \varpi_{k}>0$, the following inequality is true : $\beta_{k} \sum_{i=1}^{p} \frac{\alpha_{k_{i}}^{2}}{\varpi_{k} \gamma_{k_{i}}+1}>\beta_{k} \sum_{i=1}^{p} \frac{\alpha_{k_{i}}^{2}}{\left(\varpi_{k} \gamma_{k_{i}}+1\right)^{2}}=1$. Thus we obtain $\sigma_{k}^{2}=\sigma_{k-1}^{2}$ if $\left\|\boldsymbol{\delta}_{\boldsymbol{k}}\right\|_{V_{k}^{-1}} \leq 1$ and $\sigma_{k}^{2}<\sigma_{k-1}^{2}$ otherwise; this means that the sequence $\left(\sigma_{k}^{2}\right)_{k \in \mathbb{N}^{*}}$ is decreasing, and since it is bounded below by 0 , it is convergent on $\mathbb{R}_{+}$. This proves the point $\boldsymbol{i \boldsymbol { i }}$. of the Theorem 1 .

iii. The use of $(7 \mathrm{a}),(7 \mathrm{~b}),(7 \mathrm{~d}),(7 \mathrm{e})$ and after some manipulations, the a posteriori output error can be rewritten as

$$
\boldsymbol{y}_{\boldsymbol{k}}-F_{k} \hat{\boldsymbol{x}}_{\boldsymbol{k}}=\left(I_{p}+\omega_{k}^{*} F_{k} P_{k / k-1} F_{k}^{T} V_{k}^{-1}\right)^{-1} \boldsymbol{\delta}_{\boldsymbol{k}}
$$

the weighted norm (by $V_{k}^{-1}$ ) of which becomes, via some transformations,

$$
\left(\boldsymbol{y}_{\boldsymbol{k}}-F_{k} \hat{\boldsymbol{x}}_{\boldsymbol{k}}\right)^{T} V_{k}^{-1}\left(\boldsymbol{y}_{\boldsymbol{k}}-F_{k} \hat{\boldsymbol{x}}_{\boldsymbol{k}}\right)=\overline{\boldsymbol{\delta}}_{\boldsymbol{k}}^{T}\left(I_{p}+\omega_{k}^{*} X_{k}\right)^{-2} \overline{\boldsymbol{\delta}}_{\boldsymbol{k}}=\beta_{k} \sum_{i=1}^{p} \frac{\alpha_{k_{i}}^{2}}{\left(\omega_{k}^{*} \gamma_{k_{i}}+1\right)^{2}}= \begin{cases}1 & \text { if }\left\|\boldsymbol{\delta}_{\boldsymbol{k}}\right\|_{V_{k}^{-1}}>1, \\ \left\|\boldsymbol{\delta}_{\boldsymbol{k}}\right\|_{V_{k}^{-1}}^{2}(\leq 1) & \text { otherwise }\end{cases}
$$

$\boldsymbol{i v}$. Let $\widetilde{\varsigma}_{k}(\omega):=\sigma_{k}^{2}-\sigma_{k-1}^{2}$. Using the fact that $\overline{\boldsymbol{\delta}}_{\boldsymbol{k}}^{T}\left(\omega_{k}^{*} X_{k}+I_{p}\right)^{-1} \overline{\boldsymbol{\delta}}_{\boldsymbol{k}}=\beta_{k} \sum_{i=1}^{p} \frac{\alpha_{k_{i}}^{2}}{\omega_{k}^{*} \gamma_{k_{i}}+1}$, the point $\boldsymbol{i \boldsymbol { i }}$. allows to write

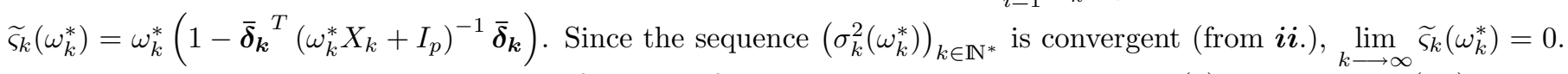
These relations require that at least one of the two following conditions holds : either (a) the sequence $\left(\omega_{k}^{*}\right)_{k \in \mathbb{N}^{*}}$ is convergent with $\lim _{k \longrightarrow \infty} \omega_{k}^{*}=0$; or (b) $\left(\overline{\boldsymbol{\delta}}_{\boldsymbol{k}}^{T}\left(\omega_{k}^{*} X_{k}+I_{p}\right)^{-1} \overline{\boldsymbol{\delta}}_{\boldsymbol{k}}\right)_{k \in \mathbb{N}^{*}}$ is convergent, with $\lim _{k \longrightarrow \infty} \overline{\boldsymbol{\delta}}_{\boldsymbol{k}}^{T}\left(\omega_{k}^{*} X_{k}+I_{p}\right)^{-1} \overline{\boldsymbol{\delta}}_{\boldsymbol{k}}=1$; 
or both. For all $k \in \mathbb{N}^{*}$ such that $\omega_{k}^{*}>0, \overline{\boldsymbol{\delta}}_{\boldsymbol{k}}^{T}\left(\omega_{k}^{*} X_{k}+I_{p}\right)^{-2} \overline{\boldsymbol{\delta}}_{\boldsymbol{k}}=1$ and $\overline{\boldsymbol{\delta}}_{\boldsymbol{k}}^{T}\left(\omega_{k}^{*} X_{k}+I_{p}\right)^{-1} \overline{\boldsymbol{\delta}}_{\boldsymbol{k}}>1$. Therefore, if the condition (a) is not satisfied, i.e., $\omega_{k}^{*} \neq 0$ when $k \mapsto \infty$, then - assuming that the limit of $\bar{\delta}_{\boldsymbol{k}}^{T}\left(\omega_{k}^{*} X_{k}+I_{p}\right)^{-1} \bar{\delta}_{k}$ exists - the condition (b) could be verified only if $\lim _{k \rightarrow \infty} \overline{\boldsymbol{\delta}}_{\boldsymbol{k}}^{T}\left(\omega_{k}^{*} X_{k}+I_{p}\right)^{-1} \overline{\boldsymbol{\delta}}_{\boldsymbol{k}}=\overline{\boldsymbol{\delta}}_{\boldsymbol{k}}^{T}\left(\omega_{k}^{*} X_{k}+I_{p}\right)^{-2} \overline{\boldsymbol{\delta}}_{\boldsymbol{k}}$; and this is true only if $\lim _{k \rightarrow \infty} \omega_{k}^{*} X_{k}=0_{p \times p}$. Now, it is shown that the conditions (9) and (10) assure that the matrix $P_{k}$ is bounded above and below (the proof is inspired from that of [16] and is omitted here for lack of place). As $F_{k}$ is a full row rank matrix and $\bar{V}_{k}$ is invertible, the boundedness of $P_{k}$ implies that there exists a positive real $\bar{c}$ such that $\forall k \in \mathbb{N}^{*}, X_{k}=\bar{V}_{k} F_{k} P_{k-1} F_{k}^{T} \bar{V}_{k}^{T} \geq \bar{c} I_{p}>0$; this shows that the condition (b) can not hold, what guarantees the satisfaction of the condition (a); the next two limits are direct consequences of this one. Indeed, by the aid of (7a), (7d) and (7b) we find $\lim _{k \longrightarrow \infty} \hat{\boldsymbol{x}}_{\boldsymbol{k}}-\hat{\boldsymbol{x}}_{\boldsymbol{k} / \boldsymbol{k}-\mathbf{1}}=\lim _{\omega \rightarrow 0} \omega P_{k / k-1} F_{k}^{T}\left(\omega F_{k} P_{k / k-1} F_{k}^{T}+V_{k}\right)^{-1} \boldsymbol{\delta}_{\boldsymbol{k}}=\mathbf{0}$;

$\lim _{k \longrightarrow \infty} P_{k}-P_{k / k-1}=\lim _{\omega \rightarrow 0}-\omega P_{k / k-1} F_{k}^{T}\left(\omega F_{k} P_{k / k-1} F_{k}^{T}+V_{k}\right)^{-1} F_{k} P_{k / k-1}=0_{n \times n}$. This proves the point $\boldsymbol{i v}$. of the theorem.

$\boldsymbol{v}$. Now, by the use of $\lim _{k \rightarrow \infty} \hat{\boldsymbol{x}}_{\boldsymbol{k}}-\hat{\boldsymbol{x}}_{\boldsymbol{k} / \boldsymbol{k}-\mathbf{1}}$ and $(7 \mathrm{e})$ it is possible to write

$\lim _{k \rightarrow \infty} \boldsymbol{\delta}_{\boldsymbol{k}}-\left(\boldsymbol{y}_{\boldsymbol{k}}-F_{k} \hat{\boldsymbol{x}}_{\boldsymbol{k}}\right)^{k \longrightarrow \infty}=\lim _{k \rightarrow \infty} F_{k}\left(\hat{\boldsymbol{x}}_{\boldsymbol{k}}-\hat{\boldsymbol{x}}_{\boldsymbol{k} / \boldsymbol{k}-\mathbf{1}}\right)=\lim _{k \rightarrow \infty} F_{k} \lim _{k \rightarrow \infty}\left(\hat{\boldsymbol{x}}_{\boldsymbol{k}}-\hat{\boldsymbol{x}}_{\boldsymbol{k} / \boldsymbol{k}-\mathbf{1}}\right)=\mathbf{0}$,

thus, $\lim _{k \rightarrow \infty} \boldsymbol{\delta}_{\boldsymbol{k}}^{T} V_{k}^{-1} \boldsymbol{\delta}_{\boldsymbol{k}}-\left(\boldsymbol{y}_{\boldsymbol{k}}-F_{k} \hat{\boldsymbol{x}}_{\boldsymbol{k}}\right)^{T} V_{k}^{-1}\left(\boldsymbol{y}_{\boldsymbol{k}}-F_{k} \hat{\boldsymbol{x}}_{\boldsymbol{k}}\right)=0$

$\Leftrightarrow \forall \varepsilon>0, \exists k_{\infty} \in \mathbb{N}^{*}, \forall k>k_{\infty}, \boldsymbol{\delta}_{\boldsymbol{k}}^{T} V_{k}^{-1} \boldsymbol{\delta}_{\boldsymbol{k}}<\left(\boldsymbol{y}_{\boldsymbol{k}}-F_{k} \hat{\boldsymbol{x}}_{\boldsymbol{k}}\right)^{T} V_{k}^{-1}\left(\boldsymbol{y}_{\boldsymbol{k}}-F_{k} \hat{\boldsymbol{x}}_{\boldsymbol{k}}\right)+\varepsilon$ and inserting the inequality $\left(\boldsymbol{y}_{\boldsymbol{k}}-F_{k} \hat{\boldsymbol{x}}_{\boldsymbol{k}}\right)^{T} V_{k}^{-1}\left(\boldsymbol{y}_{\boldsymbol{k}}-F_{k} \hat{\boldsymbol{x}}_{\boldsymbol{k}}\right) \leq 1$ (according to $\boldsymbol{i i \boldsymbol { i }}$.) achieves the proof of the point $\boldsymbol{v}$.

$\boldsymbol{v i}$. The sequence $\left(\sigma_{k}^{2}\right)_{k \in \mathbb{N}^{*}}$ is decreasing (according to $\boldsymbol{i i}$.) and, as mentioned above, the matrix $P_{k}$ is bounded below and above; it is also the case for each of its eigenvalues. Consequently, all the products $\sigma_{k}^{2} \lambda_{i}\left(P_{k}\right)(i \in\{1,2, \ldots, n\})$ corresponding to the squared semi-axes lengths of $\mathcal{E}\left(\hat{\boldsymbol{x}}_{\boldsymbol{k}}, \sigma_{k}^{2} P_{k}\right)$ are also bounded and so it is for its volume

$\operatorname{vol} \mathcal{E}\left(\hat{\boldsymbol{x}}_{\boldsymbol{k}}, \sigma_{k}^{2} P_{k}\right)=\frac{4}{3} \pi \sigma_{k}^{n} \prod_{i=1}^{n} \lambda_{i}^{\frac{1}{2}}\left(P_{k}\right)$.

vii. To prove this point, the following proposition will be applied :

Proposition $4([13])$ The system $\boldsymbol{z}_{k}=f\left(\boldsymbol{z}_{k-1}, \boldsymbol{u}_{\boldsymbol{k}-\mathbf{1}}\right)$ is ISS if it admits a continuous ISS-Lyapunov function $\mathscr{V}^{*}: \mathbb{R}^{n} \rightarrow \mathbb{R}_{+}$, that is, there exists $\mathscr{K}_{\infty}$-functions ${ }^{6} \alpha_{1}$ and $\alpha_{2}$ such that for all $\boldsymbol{z} \in \mathbb{R}^{n}$,

$\alpha_{1}(\|\boldsymbol{z}\|) \leq \mathscr{V}^{*}(\boldsymbol{z}) \leq \alpha_{2}(\|\boldsymbol{z}\|)$ and there exists a $\mathscr{K}_{\infty}$-function $\alpha_{3}$ and a $\mathscr{K}$-function $\chi$ such that for all $\boldsymbol{z} \in \mathbb{R}^{n}$ and all $\boldsymbol{u} \in \mathbb{R}^{m}, \mathscr{V}^{*}(f(\boldsymbol{z}, \boldsymbol{u}))-\mathscr{V}^{*}(\boldsymbol{z}) \leq-\alpha_{3}(\|\boldsymbol{z}\|)+\chi(\|\boldsymbol{u}\|)$.

It suffices then to show that $\mathscr{V}^{*}{ }_{k}=\widetilde{\boldsymbol{x}}_{\boldsymbol{k}}{ }^{T} P_{k}^{-1} \widetilde{\boldsymbol{x}}_{\boldsymbol{k}}$ is ISS-Lyapunov function for the error estimation system.

First, we have $\frac{\left\|\widetilde{\boldsymbol{x}}_{\boldsymbol{k}}\right\|^{2}}{\lambda_{\max }\left(P_{k}\right)} \leq \mathscr{V}^{*}{ }_{k} \leq \frac{\left\|\widetilde{\boldsymbol{x}}_{\boldsymbol{k}}\right\|^{2}}{\lambda_{\min }\left(P_{k}\right)}$.

From the point $\boldsymbol{i \boldsymbol { i }}$. of the theorem, it is clear that $-\omega \overline{\boldsymbol{\delta}}_{\boldsymbol{k}}^{T}\left(\omega_{k}^{*} X_{k}+I_{p}\right)^{-1} \overline{\boldsymbol{\delta}}_{\boldsymbol{k}}+\omega \leq 0$

Consequently, using (21) and (22), the following holds

$$
\mathscr{V}_{k}^{*}-\mathscr{V}^{*}{ }_{k / k-1} \leq \omega\left(1-\overline{\boldsymbol{\delta}}_{\boldsymbol{k}}^{T}\left(\omega_{k}^{*} X_{k}+I_{p}\right)^{-1} \overline{\boldsymbol{\delta}}_{\boldsymbol{k}}\right) \leq 0
$$

Now

$$
\mathscr{V}_{k}^{*}-\mathscr{V}^{*}{ }_{k-1} \leq \mathscr{V}_{k / k-1}^{*}-\mathscr{V}_{k-1}^{*}=-\widetilde{\boldsymbol{x}}_{\boldsymbol{k}-\mathbf{1}}{ }^{T} P_{k-1}^{-1} \widetilde{\boldsymbol{x}}_{\boldsymbol{k}-\mathbf{1}}+\widetilde{\boldsymbol{x}}_{\boldsymbol{k} / \boldsymbol{k}-\mathbf{1}}{ }^{T} P_{k / k-1}^{-1} \widetilde{\boldsymbol{x}}_{\boldsymbol{k} / \boldsymbol{k}-\mathbf{1}}
$$

where $\widetilde{\boldsymbol{x}}_{\boldsymbol{k} / \boldsymbol{k}-1}:=\boldsymbol{x}_{\boldsymbol{k}}^{*}-\hat{\boldsymbol{x}}_{\boldsymbol{k} / \boldsymbol{k}-1}=\boldsymbol{\varphi}_{\boldsymbol{k}}\left(\boldsymbol{x}_{\boldsymbol{k}-1}^{*}\right)-\boldsymbol{\varphi}_{\boldsymbol{k}}\left(\hat{\boldsymbol{x}}_{\boldsymbol{k}-1}\right)+\boldsymbol{w}_{\boldsymbol{k}-1}$ and $\widetilde{\boldsymbol{x}}_{\boldsymbol{k}-1}=\boldsymbol{x}_{\boldsymbol{k}-1}^{*}-\hat{\boldsymbol{x}}_{\boldsymbol{k}-1}$.

By using the Proposition 2 the Lemma 1 it can be proved that

$$
\begin{aligned}
\mathscr{V}_{k / k-1}^{*} \leq(1-\rho)\left(\boldsymbol{\varphi}_{\boldsymbol{k}}\left(\boldsymbol{x}_{\boldsymbol{k}-\mathbf{1}}^{*}\right)-\boldsymbol{\varphi}_{\boldsymbol{k}}\left(\hat{\boldsymbol{x}}_{\boldsymbol{k}-\mathbf{1}}\right)\right)^{T}\left(\left(\hat{\Phi}_{k-1}+\Delta_{k-1}\right)^{T} P_{k-1}\left(\hat{\Phi}_{k-1}+\Delta_{k-1}\right)\right)^{-1} \\
\times\left(\boldsymbol{\varphi}_{\boldsymbol{k}}\left(\boldsymbol{x}_{\boldsymbol{k}-\mathbf{1}}^{*}\right)-\boldsymbol{\varphi}_{\boldsymbol{k}}\left(\hat{\boldsymbol{x}}_{\boldsymbol{k}-\mathbf{1}}\right)\right)+\rho \sigma_{k-1}^{2} \boldsymbol{w}_{\boldsymbol{k}-\mathbf{1}}^{T} W_{k-1}^{-1} \boldsymbol{w}_{\boldsymbol{k}-\mathbf{1}}
\end{aligned}
$$

On the other hand, following the same reasoning that led to (16)-(17), it comes that

$$
\left(\boldsymbol{\varphi}_{\boldsymbol{k}}\left(\boldsymbol{x}_{\boldsymbol{k}-\mathbf{1}}^{*}\right)-\boldsymbol{\varphi}_{\boldsymbol{k}}\left(\hat{\boldsymbol{x}}_{\boldsymbol{k}-\mathbf{1}}\right)\right)^{T}\left(\left(\hat{\Phi}_{k-1}+\Delta_{k-1}\right)^{T} P_{k-1}\left(\hat{\Phi}_{k-1}+\Delta_{k-1}\right)\right)^{-1}\left(\boldsymbol{\varphi}_{\boldsymbol{k}}\left(\boldsymbol{x}_{\boldsymbol{k}-\mathbf{1}}^{*}\right)-\boldsymbol{\varphi}_{\boldsymbol{k}}\left(\hat{\boldsymbol{x}}_{\boldsymbol{k}-\mathbf{1}}\right)\right) \leq \widetilde{\boldsymbol{x}}_{\boldsymbol{k}-\mathbf{1}}^{T} P_{k-1}^{-1} \widetilde{\boldsymbol{x}}_{\boldsymbol{k}-\mathbf{1}}
$$

Thus

$$
\mathscr{V}_{k}^{*}-\mathscr{V}_{k-1}^{*} \leq-\rho^{*}{ }_{k-1}+\rho \sigma_{k-1}^{2} \boldsymbol{w}_{\boldsymbol{k}-\mathbf{1}}{ }^{T} W_{k-1}^{-1} \boldsymbol{w}_{\boldsymbol{k}-\mathbf{1}} \leq-\frac{\rho\left\|\widetilde{\boldsymbol{x}}_{\boldsymbol{k}-\mathbf{1}}\right\|^{2}}{\lambda_{\min }\left(P_{k-1}\right)}+\frac{\rho \sigma_{k-1}^{2}\left\|\boldsymbol{w}_{\boldsymbol{k}-\mathbf{1}}\right\|^{2}}{\lambda_{\min }\left(W_{k-1}\right)} .
$$

This means that $\mathscr{V}^{*}{ }_{k}$ is an ISS-Lyapunov function for the system which state vector is estimation error $\widetilde{\boldsymbol{x}}_{\boldsymbol{k}}$. This completes the proof of the theorem.

${ }^{6} \alpha: \mathbb{R}_{+} \rightarrow \mathbb{R}_{+}$is $\mathscr{K}_{\infty}$-function if it is a $\mathscr{K}$-function and if $\alpha(s) \rightarrow \infty$ as $s \rightarrow \infty$. 


\section{REFERENCES}

[1] F. C. Schweppe, "Recursive state estimation : unknown but bounded errors and system inputs," IEEE Transactions on Automatic Control, vol. 13, pp. 22-28, 1968.

[2] J. Norton, "Special issue on bounded-error estimation 1," International Journal of Adaptive Control and Signal Processing, vol. 8, no. 1, pp. 1-118, 1994.

[3] J. Norton, "Special issue on bounded-error estimation 2," International Journal of Adaptive Control and Signal Processing, vol. 9, no. 1, pp. 1-132, 1995

[4] S. Kapoor, S. Gollamudi, S. Nagaraj, and Y. F. Huang, "Tracking of time-varying parameters using optimal bounding ellipsoid algorithms," in Proc. $34^{\text {th }}$ Allerton Conference on Comm., Control and Computing, (Monticello, IL), 1996.

[5] D. Maksarov and J. P. Norton, "State bounding with ellipsoidal set description of the uncertainty," International Journal of Control, vol. 65 , no. 5 , pp. $847-866,1996$.

[6] C. Durieu, E. Walter, and B. Polyak, "Multi-input multi-output ellipsoidal state bounding," Journal of Optimization Theory and Applications, vol. 111, no. 2, pp. 273-303, 2001.

[7] L. Jaulin, M. Kieffer, I. Braems, and E. Walter, "Guaranteed nonlinear estimation using constraint propagation on sets," International Journal of Control, vol. 74, no. 18, pp. 1772-1782, 2001.

[8] L. Jaulin, M. Kieffer, O. Didrit, and E. Walter, Applied interval analysis with examples in parametric and state estimation, robust control and robotics. Springer, Verlag, London, 2001.

[9] A. Rapaport and H. Gouz, "Parallelotopic and practical observers for nonlinear uncertain systems," International Journal $f$ Control, vol. 76 , no. 3, 2003.

[10] E. Scholte and M. E. Campbell, "A nonlinear set-membership filter for on-line applications," International Journal of Robust and Nonlinear Control, vol. 13, pp. 1337-1358, 2003.

[11] Y. Becis-Aubry, M. Boutayeb, and M. Darouach, "A recursive algorithm for the parameter identification of mimo nonlinear models using bounding ellipsoids," in IFAC NOLCOS Symposium on Nonlinear Control Systems, (Stuttgart, Germany), 2004.

[12] Y. Becis-Aubry, M. Boutayeb, and M. Darouach, "A stable recursive filter for state estimation of linear models in the presence of bounded disturbances," in 16th IFAC World Congress, (Prague, Czech Republic), 2005.

[13] Z.-P. Jiang and Y. Wang, "Input-to-state stability for discrete-time nonlinear systems," Automatica, vol. 37, pp. 857-869, 2001.

[14] R. Marino, S. Peresada, and P. Valigi, "Adaptive Input-Output Linearizing Control of Induction Motors," IEEE Transactions on Automatic Control, vol. 38, no. 2, pp. 208-221, 1993.

[15] F. C. Schweppe, Uncertain Dynamic Systems. Prentice Hall, Englewood Cliffs, 1973.

[16] J. J. Deyst and C. F. Price, "Conditions for asymptotic stability of the discrete minimum-variance linear estimator," IEEE Transactions on Automatic Control, pp. 702-705, 1968. 